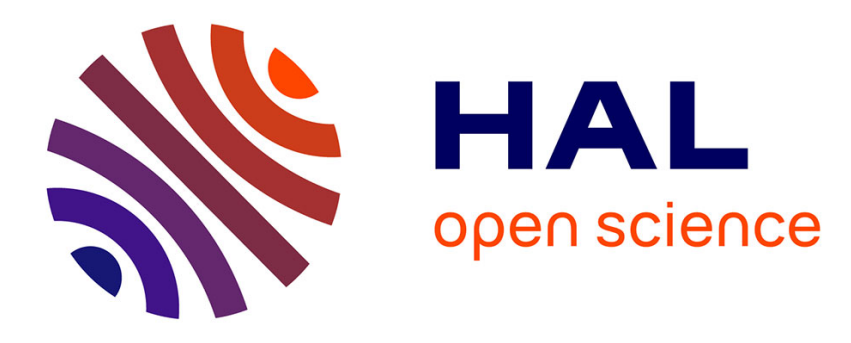

\title{
A smooth fictitious domain/multiresolution method for elliptic equations on general domains
}

Ping Yin, Jacques Liandrat

\section{To cite this version:}

Ping Yin, Jacques Liandrat. A smooth fictitious domain/multiresolution method for elliptic equations on general domains. Numerical Algorithms, 2016, 10.1007/s11075-015-0063-x . hal-01266859

\section{HAL Id: hal-01266859 \\ https://hal.science/hal-01266859}

Submitted on 3 Feb 2016

HAL is a multi-disciplinary open access archive for the deposit and dissemination of scientific research documents, whether they are published or not. The documents may come from teaching and research institutions in France or abroad, or from public or private research centers.
L'archive ouverte pluridisciplinaire HAL, est destinée au dépôt et à la diffusion de documents scientifiques de niveau recherche, publiés ou non, émanant des établissements d'enseignement et de recherche français ou étrangers, des laboratoires publics ou privés. 


\title{
A smooth fictitious domain/multiresolution method for elliptic equations on general domains
}

\author{
Ping Yin* \\ Science college, Jiangnan University, Wuxi 214122, China \\ Jacques Liandrat \\ Centrale Marseille, I2M, UMR 7373, CNRS, Aix-Marseille Univ., Marseille, 13453, \\ France
}

\begin{abstract}
We propose a smooth fictitious domain/multiresolution method for enhancing the accuracy order in solving second order elliptic partial differential equations on general bivariate domains. We prove the existence and uniqueness of the solution of corresponding discrete problem and the interior error estimate which justifies the improved accuracy order. Numerical experiments are conducted on a cassini oval.
\end{abstract}

Keywords: Elliptic partial differential equations, Smooth fictitious domain methods, Multiresolution discretization, Interior error estimate

\section{Introduction}

Recently, multiresolution methods have became a new powerful tool in the numerical approximation of partial differential equations due to their high accuracy and related fast algorithms $[1,2]$. However, it is difficult to construct scaling and wavelet functions adapted to the geometry of general domains. This is an important issue, especially in the case of moving boundary problems.

\footnotetext{
*Corresponding author

Email addresses: wanderapple@gmail.com (Ping Yin), jliandrat@centrale-marseille.fr (Jacques Liandrat)
} 
Fictitious domain methods were introduced to encounter this problem. They consist in solving a problem raised on a general domain $\omega$ by merging $\omega$ into a larger and simpler domain $\Omega$. Fictitious domain methods differ fundamentally in the way how the boundary conditions are enforced on $\gamma$, the boundary of $\omega$. In penalization methods, additional terms are added in $\Omega \backslash \omega$. These terms are usually weighted with a coefficient $\frac{1}{\epsilon}$. To the limit when $\epsilon$ goes to zero, the boundary conditions are enforced $[3,4]$. The Lagrange multipliers methods emerge from the optimization techniques under constraints. A minimization problem on a subset is transformed into a saddle point problem on a larger set. Lagrange multipliers as supplementary unknowns that may live on $\omega$, on its boundary $\gamma$ or on $\Omega \backslash \omega[5,6]$.

In this paper, the starting point is a fictitious domain method coupled with Lagrange multipliers on the boundary. By shifting the Lagrange multipliers on a control boundary $\Gamma$ located away from $\gamma$ in the outer normal direction(as proposed in [7]), this new approach is shown to preserve the good approximation properties of multiresolution methods and to improve the accuracy order of the numerical solution on $\omega$.

This work is a generalization to bivariate framework of the results presented in $[8,9]$. The paper is organized as follows. Section 2 presents the formulation of our smooth fictitious domain method and gives the approximate controllability result. Section 3 contains the multiresolution discretization and proves the existence of an unique solution of the discrete saddle point problem. Section 4 gives the main theorem of interior error estimate which theoretically justifies the improved accuracy order. We list the important aspects of numerical implementation in Section 5. Some numerical examples are presented in Section 6 to verify the accuracy of the proposed method. Section 7 concludes this paper.

\section{The smooth fictitious domain method}

We consider a Dirichlet boundary-value problem on an open, bounded domain $\omega \subset \mathbf{R}^{2}$ with Lipschitz boundary $\gamma$,

$$
\left\{\begin{aligned}
\hat{u}-\nu \Delta \hat{u} & =f & \text { in } & \omega, \\
\hat{u} & =g & \text { on } \quad & \gamma,
\end{aligned}\right.
$$

where $\nu>0, f \in L^{2}(\omega)$ and $g \in H^{\frac{1}{2}}(\gamma)$. In order to separate the difficulty of approximating the differential operator and the integration of the boundary 
condition as well as to overcome the difficulty of constructing basis functions adapted to the complicated domain $\omega$, a fictitious domain/Lagrange multiplier method (FDLM) [10] can be defined. It introduces a rectangular $\Omega$ containing $\omega, \bar{\omega} \subset \Omega$, and uses the Lagrange multipliers $\lambda$ on the boundary $\gamma$ to enforce the boundary conditions. Thus the extended problem has a solution $u$ on $\Omega$ such that $\left.u\right|_{\omega}=\hat{u}$ [11]. Since the auxiliary variables $\lambda$ represent the normal derivative jump of $u$ at $\gamma$ by green formula [12], the regularity of $u$ is limited in the vicinity of $\gamma$ such that $u \in H^{3 / 2-\epsilon}(\Omega)$, for any $\epsilon>0$. It is well known that the smoothness degree of the solution of a boundary-value problem does affect the order of convergence of a numerical solution [13]. J.Haslinger et al. [7] investigated a new formulation of fictitious domain methods connecting in putting the Lagrange multiplier $\lambda$ on a control boundary $\Gamma$ located outside of $\bar{\omega}$ to enforce the boundary condition on $\gamma$ (see in Figure 1). We will call it the smooth fictitious domain/Lagrange multiplier method (SFDLM). The Dirichlet boundary value problem (1) is

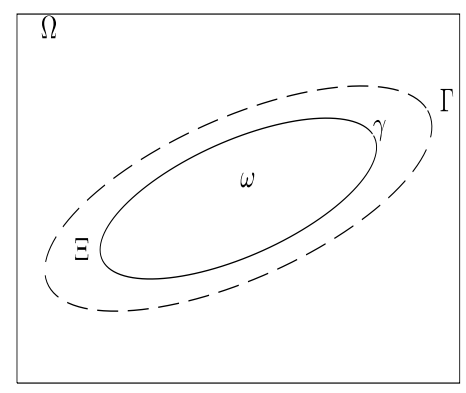

Figure 1: Geometry: $\omega$ is the original domain, $\gamma$ is its boundary. $\Gamma$ is the control boundary and $\Xi$ is the control domain with $\Xi \supset \bar{\omega}$. $\Omega$ represents the fictitious domain with $\Omega \supset \bar{\Xi}$.

then rewritten as:

$$
\left\{\begin{array}{l}
\text { Find }(u, \lambda) \in H^{1}(\Omega) \times H^{-1 / 2}(\Gamma) \text { such that } \\
a(u, v)+b_{2}(v, \lambda)=(\tilde{f}, v), \quad \forall v \in H^{1}(\Omega) \\
b_{1}(u, \mu)=<g, \mu>_{\gamma}, \quad \forall \mu \in H^{-1 / 2}(\gamma)
\end{array}\right.
$$

where, if the trace operators $B$ and $\mathcal{B}$ are defined, $B u:=\left.u\right|_{\gamma} \in H^{1 / 2}(\gamma)$, $\mathcal{B} u:=\left.u\right|_{\Gamma} \in H^{1 / 2}(\Gamma), \forall u \in H^{1}(\Omega)$ then

$$
b_{1}(u, q):=<B u, q>_{H^{1 / 2}(\gamma) \times H^{-1 / 2}(\gamma)}:=<B u, q>_{\gamma}, \forall q \in H^{-1 / 2}(\gamma),
$$




$$
b_{2}(u, p):=<\mathcal{B} u, p>_{H^{1 / 2}(\Gamma) \times H^{-1 / 2}(\Gamma)}:=<\mathcal{B} u, p>_{\Gamma}, \forall p \in H^{-1 / 2}(\Gamma) .
$$

The bilinear form $a(\cdot, \cdot)$ reads

$$
a(u, v)=\int_{\Omega}(u v+\nu \nabla u \nabla v) d x, \forall(u, v) \in H^{1}(\Omega) \times H^{1}(\Omega),
$$

and $\tilde{f}$ is an extension of $f$ from $\omega$ to $\Omega$. Note that the bilinear forms $b_{1}$ and $b_{2}$ are distinct. This kind of problem can be considered as a generalization of saddle point problems which have been studied in [14, 15]. Since the derivative jump $\lambda$ lays on $\Gamma$ at a positive distance from $\gamma$, this new method is expected to produce a smoother solution in the control domain $\Xi$ which motivates the proof of interior error estimate depending on the smoothness of $u$ in $\Xi$. For any $\lambda \in H^{-1 / 2}(\Gamma)\left(\right.$ if $H_{p}^{1}(\Omega)$ stands for the sobolev space of order 1 of periodic functions on $\Omega$ ), there exists a unique solution $\bar{u}$ of the problem $\left(2^{\prime}\right)$ :

$$
\left\{\begin{array}{l}
\text { Find } \bar{u} \in H_{p}^{1}(\Omega) \text { such that } \\
a(\bar{u}, v)=(\tilde{f}, v)-b_{2}(v, \lambda) .
\end{array}\right.
$$

However, the extended problem (2) does not have a solution in general [16]. We will use the following approximate controllability result [7].

Lemma 1. Defining the linear mapping $\Phi: H^{-1 / 2}(\Gamma) \mapsto H^{1 / 2}(\gamma)$ by $\Phi(\lambda)=$ $\left.\bar{u}\right|_{\gamma}$, where $\bar{u}$ is the solution of (2'). Then for any $\epsilon>0$, there exists a value of $\bar{\lambda}$ and the corresponding solution $\bar{u}$ associated to $\bar{g}=\Phi(\bar{\lambda})$ in (2') such that

$$
\|\hat{u}-\bar{u}\|_{H^{1}(\omega)} \leq \epsilon .
$$

\section{The multiresolution discretization}

The finite dimensional subspaces used in the numerical approximation of problem (2) include the multiresolution analysis on $\Omega, \Gamma, \gamma$ denoted by $U_{h}^{\Omega} \subset$ $H^{1}(\Omega), Q_{h^{\prime}}^{\Gamma} \subset H^{-1 / 2}(\Gamma), Q_{h^{\prime \prime}}^{\gamma} \subset H^{-1 / 2}(\gamma)$, and the subspace $V_{h}^{\Omega}$ which is the image of $U_{h}^{\Omega}$ by the operator $(I-\nu \triangle)^{-1}$, i.e., $V_{h}^{\Omega}=(I-\nu \triangle)^{-1} U_{h}^{\Omega}$. This space is spanned by biorthogonal vaguelettes [17]. We call it the Petrov-Galerkin and wavelet-vaguelette method [18]. Then the discretization formulation of (2) reads

$$
\left\{\begin{array}{l}
\text { Find }\left(u_{h}, \lambda_{h^{\prime}}\right) \in U_{h}^{\Omega} \times Q_{h^{\prime}}^{\Gamma}, \text { such that } \\
a\left(u_{h}, v_{h}\right)+b_{2}\left(v_{h}, \lambda_{h^{\prime}}\right)=\left(\tilde{f}, v_{h}\right), \quad \forall v_{h} \in V_{h}^{\Omega}, \\
b_{1}\left(u_{h}, \mu_{h^{\prime \prime}}\right)=<g, \mu_{h^{\prime \prime}}>_{\gamma}, \quad \forall \mu_{h^{\prime \prime}} \in Q_{h^{\prime \prime}}^{\gamma} .
\end{array}\right.
$$


We assume that $\Gamma$ and $\gamma$ are piecewise regular in the sequel. The definition of the various spaces reads as follows:

$U_{h}^{\Omega}=\operatorname{span}\left\{\Phi_{\alpha}^{\Omega}, \alpha \in K_{j}:=\left\{\left(j, k_{1}, k_{2}\right), 0 \leq k_{1}, k_{2} \leq 2^{j}-1,\left(j, k_{1}, k_{2}\right) \in\right.\right.$ $\left.\mathbb{N}^{3}\right\}$, where $\forall f \in L^{2}\left(\mathbb{R}^{2}\right), f_{j, k_{1}, k_{2}}(x, y)=2^{j} f\left(2^{j} x-k_{1}, 2^{j} y-k_{2}\right)$. It is an orthogonal m-order $(m \in \mathbb{N})$ multiresolution ${ }^{1}$ on $\Omega$ with $h=2^{-j}$ (see [17]). The basis function $\Phi_{\alpha}^{\Omega}$ is the tensor product of $\phi_{j, k_{1}}^{\left[0, L_{1}\right]}(x)$ and $\phi_{j, k_{2}}^{\left[0, L_{2}\right]}(y)$ with $\Omega=\left[0, L_{1}\right] \times\left[0, L_{2}\right]$, where $\phi_{j, k_{1}}^{\left[0, L_{1}\right]}(x)\left(\phi_{j, k_{2}}^{\left[0, L_{2}\right]}(y)\right)$ is the scaling function on the interval $\left[0, L_{1}\right]\left(\operatorname{resp}\left[0, L_{2}\right]\right)$ in the $\mathrm{x}(\operatorname{resp} \mathrm{y})$ direction.

$V_{h}^{\Omega}=\operatorname{span}\left\{\theta_{\alpha}^{\Omega}, \alpha \in K_{j}\right\} . \theta_{\alpha}^{\Omega}=(I-\nu \triangle)^{-1} \Phi_{\alpha}^{\Omega}$. For any $\epsilon$, there exists an integer $r>0$ and $g \in U_{\tilde{h}}^{\Omega}, \tilde{h}=2^{-j-r}$ such that $\left\|\theta_{\alpha}^{\Omega}-g\right\| \leq \epsilon$ [19]. Thus we can approximate $\theta_{\alpha}^{\Omega}$ by an element in $U_{\tilde{h}}^{\Omega}$ up to arbitrary precision.

$Q_{h^{\prime}}^{\Gamma}=\operatorname{span}\left\{\phi_{\alpha^{\prime}}^{\Gamma}, \alpha^{\prime} \in K_{j}^{\prime}:=\left\{\left(j^{\prime}, k^{\prime}\right),\left(j^{\prime}, k^{\prime}\right) \in \mathbb{N}^{2}\right\}\right\}$. It is an orthogonal $\mathrm{m}^{\prime}-\operatorname{order}\left(m^{\prime} \in \mathbb{N}\right)$ multiresolution on $\Gamma$ constructed by a map from the multiresolution on $[0,1]$ with $h^{\prime}=2^{-j^{\prime}}[20]$.

$\left.Q_{h^{\prime \prime}}^{\gamma}=\operatorname{span}\left\{\phi_{\alpha^{\prime \prime}}^{\gamma}, \alpha^{\prime \prime} \in K_{j}^{\prime \prime}:=\left\{\left(j^{\prime \prime}, k^{\prime \prime}\right),\left(j^{\prime \prime}, k^{\prime \prime}\right) \in \mathbb{N}^{2}\right\}\right\}\right\}$. It is an orthogonal m"-order $\left(m^{\prime \prime} \in \mathbb{N}\right)$ multiresolution on $\gamma$ with $h^{\prime \prime}=2^{-j^{\prime \prime}}$.

The matrix form of (4) is

$$
\left\{\begin{array}{l}
\text { Find } U_{h} \in \mathbf{R}^{4^{j}}, \Lambda_{h^{\prime}} \in \mathbf{R}^{2^{j^{\prime}}}, \text { such that } \\
\left(\begin{array}{cc}
I & C \\
D & 0
\end{array}\right)\left(\begin{array}{c}
U_{h} \\
\Lambda_{h^{\prime}}
\end{array}\right)=\left(\begin{array}{c}
F \\
G
\end{array}\right)
\end{array}\right.
$$

where the off-diagonal matrices

$$
C_{\alpha, \alpha^{\prime}}=\int_{\Gamma} \theta_{\alpha}^{\Omega} \phi_{\alpha^{\prime}}^{\Gamma} d s, D_{\alpha^{\prime \prime}, \alpha}=\int_{\gamma} \Phi_{\alpha}^{\Omega} \phi_{\alpha^{\prime \prime}}^{\gamma} d s,
$$

and the vectors at the right side

$$
(F)_{\alpha}=\int_{\Omega} \tilde{f} \theta_{\alpha}^{\Omega} d x,(G)_{\alpha^{\prime \prime}}=\int_{\gamma} g \phi_{\alpha^{\prime \prime}}^{\gamma} d s .
$$

Assuming the discretization level $j^{\prime}$ and $j^{\prime \prime}$ are equal, the matrix involved in (5)

$$
\mathcal{A}:=\left(\begin{array}{cc}
I & C \\
D & 0
\end{array}\right)
$$

\footnotetext{
${ }^{1}$ The multiresolution is of order $m$ if it produces polynomials of degree up to $m-1$.
} 
is square. To prove that there exists a unique solution of (4), we only need to show that $\mathcal{A}$ is invertible. We will use the following lemma [21].

Lemma 2. $\mathcal{A}$ is invertible if and only if $C$ has a full-column rank and

$$
\operatorname{Ker}(D) \cap R(C)=\{0\}
$$

where $\operatorname{Ker}(D)$ is the null space of matrix $D$ and $R(C)$ is the range space of matrix $C$.

Therefore, if the conditions in Lemma 2 are satisfied, (4) has a unique solution.

Theorem 3. Let $U_{h}^{\Omega}, V_{h}^{\Omega}, Q_{h^{\prime}}^{\Gamma}$ and $Q_{h^{\prime \prime}}^{\gamma}$ be constructed as previously. If $Q_{h^{\prime}}^{\Gamma} \in H^{s^{\prime}-\frac{1}{2}}(\Gamma)$ with $s^{\prime}>2, Q_{h^{\prime \prime}}^{\gamma} \in H^{s^{\prime \prime}-\frac{1}{2}}(\gamma)$ with $s^{\prime \prime}>1$ such that $j\left(s^{\prime}-\right.$ $2)-j^{\prime}\left(s^{\prime}-1\right)>B$ where $B$ is a positive constant, then there exists a unique solution of (4).

Proof. We mimic the proof of Theorem 3.1 in [22]. The condition $j\left(s^{\prime}-\right.$ $2)-j^{\prime}\left(s^{\prime}-1\right)>B$ ensures that the following LBB condition holds [23], i.e., there exist constants $\beta_{1}, \beta_{2}$ such that

$$
\begin{aligned}
& \sup _{\substack{v_{h} \in V_{h}^{\Omega} \\
v_{h} \neq 0}} \frac{b_{2}\left(v_{h}, p_{h^{\prime}}\right)}{\left\|v_{h}\right\|_{H^{2}(\Omega)}} \geq \beta_{1}\left\|p_{h^{\prime}}\right\|_{H^{-\frac{1}{2}}(\Gamma)}, \quad \forall p_{h^{\prime}} \in Q_{h^{\prime}}^{\Gamma}, \\
& \sup _{\substack{u_{h} \in U_{h}^{\Omega} \\
u_{h} \neq 0}} \frac{b_{1}\left(u_{h}, \tilde{q}_{h^{\prime \prime}}\right)}{\left\|u_{h}\right\|_{H^{1}(\Omega)}} \geq \beta_{2}\left\|\tilde{q}_{h^{\prime \prime}}\right\|_{H^{-\frac{1}{2}}(\gamma)}, \quad \forall \tilde{q}_{h^{\prime \prime}} \in Q_{h^{\prime \prime}}^{\gamma} .
\end{aligned}
$$

We then estimate the condition number of $C^{T} C, D^{T} D$ and $(D C)^{T} D C$ as follows.

-estimation of $C^{T} C$

According to the definition of the matrix $C$, it follows

$$
\begin{aligned}
\forall w \in \mathbb{R}^{2^{j^{\prime}}},\left(C^{T} C w, w\right) & =\sum_{\alpha \in K_{j}}\left(\sum_{\alpha^{\prime} \in K_{j}^{\prime}} C_{\alpha, \alpha^{\prime}} w_{\alpha^{\prime}}\right)^{2} \\
& =\sum_{\alpha \in K_{j}}\left|\int_{\Gamma} \theta_{\alpha}^{\Omega} \sum_{\alpha^{\prime} \in K_{j}^{\prime}} w_{\alpha^{\prime}} \phi_{\alpha^{\prime}}^{\Gamma}\right|^{2} .
\end{aligned}
$$


Substituting $v_{h}=\sum_{\alpha \in K_{j}} z_{\alpha} \theta_{\alpha}^{\Omega}, p_{h^{\prime}}=\sum_{\alpha^{\prime} \in K_{j}^{\prime}} w_{\alpha^{\prime}} \phi_{\alpha^{\prime}}^{\Gamma}$ in (6), we have

$$
\sup _{z \in \mathbb{R}^{4 j}} \frac{\int_{\Gamma} \sum_{\alpha \in K_{j}} z_{\alpha} \theta_{\alpha}^{\Omega} \sum_{\alpha^{\prime} \in K_{j}^{\prime}} w_{\alpha^{\prime}} \phi_{\alpha^{\prime}}^{\Gamma}}{\left\|\sum_{\alpha \in K_{j}} z_{\alpha} \theta_{\alpha}^{\Omega}\right\|_{H^{2}(\Omega)}} \geq \beta_{1}\left\|\sum_{\alpha^{\prime} \in K_{j}^{\prime}} w_{\alpha^{\prime}} \phi_{\alpha^{\prime}}^{\Gamma}\right\|_{H^{-1 / 2}(\Gamma)}, \forall w_{\alpha^{\prime}} \in \mathbb{R}^{2^{j^{\prime}}} .
$$

By the discrete Cauchy-Schwartz inequality, it yields

$$
\int_{\Gamma} \sum_{\alpha \in K_{j}} z_{\alpha} \theta_{\alpha}^{\Omega} \sum_{\alpha^{\prime} \in K_{j}^{\prime}} w_{\alpha^{\prime}} \phi_{\alpha^{\prime}}^{\Gamma} \leq(z, z)^{1 / 2}\left(C^{T} C w, w\right)^{1 / 2} .
$$

Using this inequality, it leads to

$$
\begin{aligned}
\beta_{1}\left\|\sum_{\alpha^{\prime} \in K_{j}^{\prime}} w_{\alpha^{\prime}} \phi_{\alpha^{\prime}}^{\Gamma}\right\|_{H^{-1 / 2}(\Gamma)} & \leq \sup _{z \in \mathbb{R}^{4 j}} \frac{\int_{\Gamma} \sum_{\alpha \in K_{j}} z_{\alpha} \theta_{\alpha}^{\Omega} \sum_{\alpha^{\prime} \in K_{j}^{\prime}} w_{\alpha^{\prime}} \phi_{\alpha^{\prime}}^{\Gamma}}{\left\|\sum_{\alpha \in K_{j}} z_{\alpha} \theta_{\alpha}^{\Omega}\right\|_{H^{2}(\Omega)}} \\
& \leq \frac{(z, z)^{1 / 2}}{\left\|\sum_{\alpha \in K_{j}} z_{\alpha} \theta_{\alpha}^{\Omega}\right\|_{H^{2}(\Omega)}}\left(C^{T} C w, w\right)^{1 / 2} .
\end{aligned}
$$

Due to $\theta_{\alpha}^{\Omega}=(I-\nu \triangle)^{-1} \Phi_{\alpha}^{\Omega}$ and the orthonormality of the bases $\left\{\Phi_{\alpha}^{\Omega}\right\}_{\alpha}$ in the norm $\|\cdot\|_{L^{2}(\mathbb{R})}$, it follows

$$
\left\|\sum_{\alpha \in K_{j}} z_{\alpha} \theta_{\alpha}^{\Omega}\right\|_{H^{2}(\Omega)} \sim\left\|\sum_{\alpha \in K_{j}} z_{\alpha} \Phi_{\alpha}^{\Omega}\right\|_{L^{2}(\Omega)}=(z, z)^{1 / 2} .
$$

Applying the Bernstein inequality[24] on the left term, we have

$$
\left\|\sum_{\alpha^{\prime} \in K_{j}^{\prime}} w_{\alpha^{\prime}} \phi_{\alpha^{\prime}}^{\Gamma}\right\|_{H^{-1 / 2}(\Gamma)} \geq c 2^{-j^{\prime} / 2}(w, w)^{1 / 2}
$$

where the constant $C>0$ depends on the property of the multiresolution on the boundary $\Gamma$.

Combing the (8),(9) and (9) together, it follows

$$
\left(C^{T} C w, w\right) \geq K_{1, c} 2^{-j^{\prime}}(w, w), \forall w \in \mathbb{R}^{2^{j^{\prime}}} .
$$

On the other hand, we use the continuous Cauchy-Schwartz inequality to get 


$$
\begin{aligned}
\int_{\Gamma} \sum_{\alpha \in K_{j}} z_{\alpha} \theta_{\alpha}^{\Omega} \sum_{\alpha^{\prime} \in K_{j}^{\prime}} w_{\alpha^{\prime}} \phi_{\alpha^{\prime}}^{\Gamma} & \leq\left\|\sum_{\alpha \in K_{j}} z_{\alpha} \theta_{\alpha}^{\Omega}\right\|_{L^{2}(\Gamma)}\left\|\sum_{\alpha^{\prime} \in K_{j}^{\prime}} w_{\alpha^{\prime}} \phi_{\alpha^{\prime}}^{\Gamma}\right\|_{L^{2}(\Gamma)} \\
& \leq C\left\|\sum_{\alpha \in K_{j}} z_{\alpha} \theta_{\alpha}^{\Omega}\right\|_{H^{1}(\Omega)}(w, w)^{1 / 2} \\
& \leq C(z, z)^{1 / 2}(w, w)^{1 / 2}
\end{aligned}
$$

where $C>0$ only denotes a constant, does not mean the same value.

Choosing $z_{\alpha}=\int_{\Gamma} \theta_{\alpha}^{\Omega} \sum_{\alpha^{\prime} \in K_{j}^{\prime}} w_{\alpha^{\prime}} \phi_{\alpha^{\prime}}^{\Gamma}$, then we have

$$
\left(C^{T} C w, w\right) \leq K_{2, c}(w, w) \text {. }
$$

Therefore,

$$
K_{1, c} 2^{-j^{\prime}}(w, w) \leq\left(C^{T} C w, w\right) \leq K_{2, c}(w, w) .
$$

-estimation of $D^{T} D$

We use the definition of the matrix $D$ to get

$$
\begin{aligned}
\forall z \in \mathbb{R}^{4^{j}},\left(D^{T} D z, z\right) & =\sum_{\alpha^{\prime \prime} \in K_{j}^{\prime \prime}}\left(\sum_{\alpha \in K_{j}} D_{\alpha^{\prime \prime}, \alpha} z_{\alpha}\right)^{2} \\
& =\sum_{\alpha^{\prime \prime} \in K_{j}^{\prime \prime}}\left|\int_{\gamma} \phi_{\alpha^{\prime \prime}}^{\gamma} \sum_{\alpha \in K_{j}} z_{\alpha} \Phi_{\alpha}^{\Omega}\right|^{2} .
\end{aligned}
$$

Taking $u_{h}=\sum_{\alpha \in K_{j}} z_{\alpha} \Phi_{\alpha}^{\Omega}, \tilde{q}_{h^{\prime \prime}}=\sum_{\alpha^{\prime \prime} \in K_{j}^{\prime \prime}} w_{\alpha^{\prime \prime}} \phi_{\alpha^{\prime \prime}}^{\gamma}$ in (7), it follows

$$
\sup _{z \in \mathbb{R}^{4^{j}}} \frac{\int_{\gamma} \sum_{\alpha \in K_{j}} z_{\alpha} \Phi_{\alpha}^{\Omega} \sum_{\alpha^{\prime \prime} \in K_{j}^{\prime \prime}} w_{\alpha^{\prime \prime}} \phi_{\alpha^{\prime \prime}}^{\gamma}}{\left\|\sum_{\alpha \in K_{j}} z_{\alpha} \Phi_{\alpha}^{\Omega}\right\|_{H^{1}(\Omega)}} \geq \beta_{2}\left\|\sum_{\alpha^{\prime \prime} \in K_{j}^{\prime \prime}} w_{\alpha^{\prime \prime}} \phi_{\alpha^{\prime \prime}}^{\gamma}\right\|_{H^{-1 / 2}(\gamma)}
$$

By the discrete Cauchy-Schartz inequality, it follows

$$
\int_{\gamma} \sum_{\alpha \in K_{j}} z_{\alpha} \Phi_{\alpha}^{\Omega} \sum_{\alpha^{\prime \prime} \in K_{j}^{\prime \prime}} w_{\alpha^{\prime \prime}} \phi_{\alpha^{\prime \prime}}^{\gamma} \leq(w, w)^{1 / 2}\left(D^{T} D z, z\right)^{1 / 2} .
$$

Using (12) and the Bernstein inequality of the multiresolution $Q_{h^{\prime \prime}}^{\gamma}$, we have

$$
\begin{aligned}
\left(D^{T} D z, z\right)^{1 / 2} & \geq \beta_{2} 2^{-j^{\prime \prime} / 2}\left\|\sum_{\alpha \in K_{j}} z_{\alpha} \Phi_{\alpha}^{\Omega}\right\|_{H^{1}(\Omega)} \\
& \geq \beta_{2} 2^{-j^{\prime \prime} / 2}\left\|\sum_{\alpha \in K_{j}} z_{\alpha} \Phi_{\alpha}^{\Omega}\right\|_{L^{2}(\Omega)} \\
& =\beta_{2} 2^{-j^{\prime \prime} / 2}(z, z)^{1 / 2} .
\end{aligned}
$$


The inequality above equals to

$$
\left(D^{T} D z, z\right) \geq K_{1, D} 2^{-j^{\prime \prime}}(z, z) .
$$

By the continuous Cauchy-Schwartz inequality, it follows

$$
\begin{aligned}
\int_{\gamma} \sum_{\alpha \in K_{j}} z_{\alpha} \Phi_{\alpha}^{\Omega} \sum_{\alpha^{\prime \prime} \in K_{j}^{\prime \prime}} w_{\alpha^{\prime \prime}} \phi_{\alpha^{\prime \prime}}^{\gamma} & \leq\left\|\sum_{\alpha \in K_{j}} z_{\alpha} \Phi_{\alpha}^{\Omega}\right\|_{L^{2}(\gamma)}\left\|\sum_{\alpha^{\prime \prime} \in K_{j}^{\prime \prime}} w_{\alpha^{\prime \prime}} \phi_{\alpha^{\prime \prime}}^{\gamma}\right\|_{L^{2}(\gamma)} \\
& \leq C\left\|\sum_{\alpha \in K_{j}} z_{\alpha} \Phi_{\alpha}^{\Omega}\right\|_{H^{1}(\Omega)}(w, w)^{1 / 2} \\
& \leq C 2^{j}(z, z)^{1 / 2}(w, w)^{1 / 2}
\end{aligned}
$$

where we use the Bernstein inequality of the multiresolution $U_{h}^{\Omega}$ and the trace theorem.

Choosing $w_{\alpha^{\prime \prime}}=\int_{\gamma} \phi_{\alpha^{\prime \prime}}^{\gamma} \sum_{\alpha \in K_{j}} z_{\alpha} \Phi_{\alpha}^{\Omega}$, we get

$$
\left(D^{T} D z, z\right) \leq K_{2, D} 2^{2 j}(z, z)
$$

Therefore,

$$
K_{1, D} 2^{-j^{\prime \prime}}(z, z) \leq\left(D^{T} D z, z\right) \leq K_{2, D} 2^{2 j}(z, z) .
$$

-estimation of $(D C)^{T} D C$

Taking $z=c w$ in (13), we have

$$
2^{-j^{\prime \prime}} K_{1, D}(C w, C w) \leq\left(D^{T} D C w, C w\right) \leq 2^{2 j} K_{2, D}(C w, C w) .
$$

By (11), it yields

$$
2^{-\left(j^{\prime}+j^{\prime \prime}\right)} K_{1, D} K_{1, C}(w, w) \leq\left(D^{T} D C w, C w\right) \leq 2^{2 j} K_{2, D} K_{2, C}(w, w) .
$$

Denote $K_{1}=K_{1, D} K_{1, C}, k_{2}=K_{2, D} K_{2, C}$, and here $j^{\prime}=j^{\prime \prime}$, we have

$$
K_{1} 2^{-2 j^{\prime}}(w, w) \leq(D C w, D C w) \leq K_{2} 2^{2 j}(w, w), \forall w \in \mathbb{R}^{2^{j^{\prime}}},
$$

Thus it yields that $\mathrm{C}$ has a full-column rank and $\operatorname{Ker}(D) \cap \mathrm{R}(C)=\{0\}$. By Lemma 2, the proof concludes. 


\section{Interior error estimate}

We will use the notation $A \lesssim B$ which means that there exits a constant $c>0$ such that $A \leq c B$, similarly for $\gtrsim$. Local interior error estimate deals with the error between the computed solution $u_{h}$ and $\bar{u}$, a good approximation of $u$ on $\omega$, on $\square$ a subdomain of $\Xi$ including $\bar{\omega}$. This estimate depends on the regularity of $\bar{u}$. We first recall the results for the bilinear form $a(\cdot, \cdot)$ $[22]$.

Lemma 4. For $U_{h}^{\Omega}, V_{h}^{\Omega}$ constructed as previously, there exist

$$
\begin{aligned}
& \sup _{\substack{v_{h} \in V_{h}^{\Omega} \\
v_{h} \neq 0}} \frac{a\left(u_{h}, v_{h}\right)}{\left\|v_{h}\right\|_{H^{1}(\Omega)}} \gtrsim\left\|u_{h}\right\|_{H^{1}(\Omega)}, \quad \forall u_{h} \in U_{h}^{\Omega}, \\
& \sup _{\substack{u_{h} \in U_{h}^{\Omega} \\
u_{h} \neq 0}} \frac{a\left(u_{h}, v_{h}\right)}{\left\|u_{h}\right\|_{H^{1}(\Omega)}} \gtrsim\left\|v_{h}\right\|_{H^{1}(\Omega)}, \quad \forall v_{h} \in V_{h}^{\Omega} .
\end{aligned}
$$

Then, we derive the interior error estimate as follows.

Theorem 5. Let $\omega \subset \subset \square \subset \subset \Xi \subset \subset \Omega, \hat{u}$ (resp $u_{h}$ ) be the solution of problem of (1) (resp problem (4)). Moreover, $(\bar{u}, \bar{\lambda})$ is the solution of problem (2') associated to $\bar{g}=\Phi(\bar{\lambda})$ such that $\bar{u}$ satisfies (3). Then if $\bar{u} \in H^{s}(\square)$, $1<s \leq m, \bar{\lambda} \in H^{s^{\prime}}(\Gamma), 0<s^{\prime} \leq m^{\prime}$, and $j \geq j_{0}\left(j_{0}\right.$ depending on $\omega$ and $\left.\Xi\right)$, we have

$$
\left\|\hat{u}-u_{h}\right\|_{H^{1}(\omega)} \lesssim 2^{-j(s-1)}\|\bar{u}\|_{H^{s}(\square)}+2^{-j^{\prime} s^{\prime}}\|\bar{\lambda}\|_{H^{s^{\prime}}(\Gamma)}+\epsilon, \forall \epsilon>0 .
$$

Proof. Let $\omega \subset \subset \square_{0} \subset \subset \square \subset \subset \Xi \subset \subset \Omega$ and $\delta=1$ on $\omega, \delta \in C_{0}^{\infty}\left(\square_{0}\right)$ and set $\tilde{u}=\delta \bar{u}$. Let $T \tilde{u} \in U_{h}^{\Omega}$ be the unique solution of

$$
a\left(\tilde{u}-T \tilde{u}, v_{h}\right)=-b_{2}\left(v_{h}, \bar{\lambda}-\tilde{\lambda}\right), \quad \forall v_{h} \in V_{h}^{\Omega},
$$

where $\tilde{\lambda} \in Q_{h^{\prime}}^{\Gamma}$ solves $\|\tilde{\lambda}-\bar{\lambda}\|_{H^{-1 / 2}(\Gamma)}=\inf _{\lambda_{h^{\prime}} \in Q_{h^{\prime}}^{\Gamma}}\left\|\lambda_{h^{\prime}}-\bar{\lambda}\right\|_{H^{-1 / 2}(\Gamma)}$. The existence of $T \tilde{u}$ is guaranteed by Lemma 4 and Lax-Milgram lemma.

Now

$$
\begin{aligned}
\|\tilde{u}-T \tilde{u}\|_{H^{1}(\Omega)} & \lesssim \sup _{v \in H^{1}(\Omega)} \frac{a(\tilde{u}-T \tilde{u}, v)}{\|v\|_{H^{1}(\Omega)}} \\
& \lesssim \sup _{v \in H^{1}(\Omega)}\left(\frac{a\left(\tilde{u}-T \tilde{u}, v-P^{*} v\right)}{\|v\|_{H^{1}(\Omega)}}-\frac{b_{2}\left(P^{*} v, \bar{\lambda}-\tilde{\lambda}\right)}{\|v\|_{H^{1}(\Omega)}}\right),
\end{aligned}
$$


where $P^{*} v \in V_{h}^{\Omega}$ satisfies

$$
a\left(u_{h}, v-P^{*} v\right)=0, \quad \forall u_{h} \in U_{h}^{\Omega} .
$$

Furthermore, it follows from (15) that

$$
\left\|P^{*} v\right\|_{H^{1}(\Omega)} \lesssim \sup _{u_{h} \in U_{h}^{\Omega}} \frac{a\left(u_{h}, P^{*} v\right)}{\left\|u_{h}\right\|_{H^{1}(\Omega)}}=\sup _{u_{h} \in U_{h}^{\Omega}} \frac{a\left(u_{h}, v\right)}{\left\|u_{h}\right\|_{H^{1}(\Omega)}} \lesssim\|v\|_{H^{1}(\Omega)} .
$$

Hence, for any $\eta \in U_{h}^{\Omega}$,

$$
a\left(\tilde{u}-T \tilde{u}, v-P^{*} v\right)=a\left(\tilde{u}-\eta, v-P^{*} v\right) \lesssim\|\tilde{u}-\eta\|_{H^{1}(\Omega)}\|v\|_{H^{1}(\Omega)},
$$

where we have used (18). Also

$$
\begin{aligned}
\left|b_{2}\left(P^{*} v, \bar{\lambda}-\tilde{\lambda}\right)\right| & =\left|\left(\mathcal{B} P^{*} v, \bar{\lambda}-\tilde{\lambda}\right)_{\Gamma}\right| \lesssim\left\|P^{*} v\right\|_{H^{1}(\Omega)}\|\bar{\lambda}-\tilde{\lambda}\|_{H^{-1 / 2}(\Gamma)} \\
& \lesssim\|v\|_{H^{1}(\Omega)} 2^{-j^{\prime} s^{\prime}}\|\bar{\lambda}\|_{H^{s^{\prime}}(\Gamma)},
\end{aligned}
$$

where the trace theorem and Jackson estimate are used.

It follows from (17), (19) and (20) that

$$
\begin{aligned}
\|\tilde{u}-T \tilde{u}\|_{H^{1}(\Omega)} & \lesssim \inf _{\eta \in U_{h}^{\Omega}}\|\tilde{u}-\eta\|_{H^{1}(\Omega)}+2^{-j^{\prime} s^{\prime}}\|\bar{\lambda}\|_{H^{s^{\prime}(\Gamma)}} \\
& \lesssim 2^{-j(s-1)}\|\tilde{u}\|_{H^{s}(\square)}+2^{-j^{\prime} s^{\prime}}\|\bar{\lambda}\|_{H^{s^{\prime}(\Gamma)}} \\
& \lesssim 2^{-j(s-1)}\|\bar{u}\|_{H^{s}(\square)}+2^{-j^{\prime} s^{\prime}}\|\bar{\lambda}\|_{H^{s^{\prime}(\Gamma)}} .
\end{aligned}
$$

Hence,

$$
\begin{aligned}
\|\bar{u}-T \tilde{u}\|_{H^{1}(\omega)} & =\|\tilde{u}-T \tilde{u}\|_{H^{1}(\omega)} \leq\|\tilde{u}-T \tilde{u}\|_{H^{1}(\Omega)} \\
& \lesssim 2^{-j(s-1)}\|\bar{u}\|_{H^{s}(\square)}+2^{-j^{\prime} s^{\prime}}\|\bar{\lambda}\|_{H^{s^{\prime}(\Gamma)}} .
\end{aligned}
$$

Let us now estimate $\left\|\hat{u}-u_{h}\right\|_{H^{1}(\omega)}$.

$$
\begin{aligned}
\left\|\hat{u}-u_{h}\right\|_{H^{1}(\omega)} & \leq\|\hat{u}-\bar{u}\|_{H^{1}(\omega)}+\left\|\bar{u}-u_{h}\right\|_{H^{1}(\omega)} \\
& \leq \epsilon+\|\bar{u}-T \tilde{u}\|_{H^{1}(\omega)}+\left\|T \tilde{u}-u_{h}\right\|_{H^{1}(\omega)} .
\end{aligned}
$$

Note $(\bar{u}, \bar{\lambda})\left(\operatorname{resp}\left(u_{h}, \lambda_{h^{\prime}}\right)\right)$ is the solution of (2') (resp (4)), we subtract these two equations to have

$$
a\left(\bar{u}-u_{h}, v_{h}\right)+b_{2}\left(v_{h}, \bar{\lambda}-\lambda_{h^{\prime}}\right)=0, \quad \forall v_{h} \in V_{h}^{\Omega} .
$$


It follows then from (16) that

$$
a\left(T \tilde{u}-u_{h}, v_{h}\right)=a\left(\tilde{u}-\bar{u}, v_{h}\right)+b_{2}\left(v_{h}, \lambda_{h^{\prime}}-\tilde{\lambda}\right), \quad \forall v_{h} \in V_{h}^{\Omega} .
$$

Let $P_{V_{h}^{\Omega}}$ be the biorthogonal projector on $V_{h}^{\Omega}[22]$, we have

$$
a\left(u_{h}, P_{V_{h}^{\Omega}} v\right)=a\left(u_{h}, v\right), \forall\left(u_{h}, v\right) \in U_{h}^{\Omega} \times H^{1}(\Omega),
$$

and

$$
\left\|P_{V_{h}^{\Omega}} v\right\|_{H^{1}(\square)} \lesssim\|v\|_{H^{1}(\square)}, \quad \forall v \in H_{0}^{1}(\square)
$$

Hence,

$$
\begin{aligned}
\left\|T \tilde{u}-u_{h}\right\|_{H^{1}(\omega)} & \leq\left\|T \tilde{u}-u_{h}\right\|_{H^{1}\left(\square_{0}\right)} \lesssim \sup _{v \in H_{0}^{1}(\square)} \frac{a\left(T \tilde{u}-u_{h}, v\right)}{\|v\|_{H^{1}(\square)}} \\
& \lesssim \sup _{v \in H_{0}^{1}(\square)} \frac{a\left(T \tilde{u}-u_{h}, P_{\left.V_{h}^{\Omega} v\right)}\right.}{\left\|P_{V_{h}^{\Omega}} v\right\|_{H^{1}(\square)}} .
\end{aligned}
$$

If the scale level $j$ is sufficiently large, then $P_{V_{h}^{\Omega}} v \in \stackrel{\circ}{V}_{h}^{\Omega}(\square):=\left\{v_{h} \mid v_{h} \in\right.$ $\left.V_{h}^{\Omega}, \operatorname{supp} v_{h} \subset \square\right\}, \forall v \in H_{0}^{1}(\square)$. It follows from (23) and (24) that

$$
\begin{aligned}
\left\|T \tilde{u}-u_{h}\right\|_{H^{1}(\omega)} & \lesssim \sup _{v_{h} \in \dot{V}_{h}^{\Omega}(\square)} \frac{a\left(T \tilde{u}-u_{h}, v_{h}\right)}{\left\|v_{h}\right\|_{H^{1}(\square)}}=\sup _{v_{h} \in \dot{V}_{h}^{\Omega}(\square)} \frac{a\left(\tilde{u}-\bar{u}, v_{h}\right)}{\left\|v_{h}\right\|_{H^{1}(\square)}} \\
& \lesssim \inf _{\eta \in U_{h}^{\Omega}}\|\tilde{u}-\eta\|_{H^{1}(\square)}+\inf _{\eta \in U_{h}^{\Omega}}\|\bar{u}-\eta\|_{H^{1}(\square)} \\
& \lesssim 2^{-j(s-1)}\|\bar{u}\|_{H^{s}(\square) .}
\end{aligned}
$$

By (21), (22) and (25), we have

$$
\left\|\hat{u}-u_{h}\right\|_{H^{1}(\omega)} \lesssim 2^{-j(s-1)}\|\bar{u}\|_{H^{s}(\square)}+2^{-j^{\prime} s^{\prime}}\|\bar{\lambda}\|_{H^{s^{\prime}(\Gamma)}}+\epsilon,
$$

which completes the proof.

Here the second term in the right hand side of the estimate of Theorem 5 illustrates the influence of the Lagrange multiplier, i.e., the amplitude of the normal gradient jump at the control boundary. 


\section{Numerical implementation}

\subsection{Point value of $\Phi_{\alpha}^{\Omega}$ and $\theta_{\alpha}^{\Omega}$}

Assuming the order $\mathrm{m}$ of the spline multiresolution on $\Omega$ is even, the orthonormal scaling function $\Phi_{\alpha}^{\Omega}$ is derived from the tensor product of $\phi_{j, k_{1}}^{\left[0, L_{1}\right]}(x)$ and $\phi_{j, k_{2}}^{\left[0, L_{2}\right]}(y)$ which are periodic as constructed in [25]. For simplicity, we assume that $L_{1}=L_{2}=1$, i.e., $\Omega=[0,1] \times[0,1]$. We write $\phi_{j, k}^{[0,1]}$ as a linear combination of B-spline denoted by $N_{m}\left(N_{m}=(*)^{m} \chi_{[0,1]}\right)$, seen in [8],

$$
\phi_{j, k}(x)=\phi_{j, 0}\left(x-k / 2^{j}\right)=2^{j / 2} \sum_{k=-m / 2}^{2^{j}+m / 2} b_{k} N_{m}\left(2^{j} x-2 k+m / 2\right) .
$$

Note $\theta_{\alpha}^{\Omega}$ can be approximated by an element in space $V_{j^{\prime}}\left(j^{\prime}=j+r\right.$ up to arbitrary precision, $r \in \mathbf{N}^{+}$), i.e.,

$$
\theta_{\alpha}^{\Omega} \sim \sum_{\alpha^{\prime} \in K_{j^{\prime}}} C_{\alpha^{\prime}}^{\alpha} \Phi_{\alpha^{\prime}}^{\Omega}
$$

where $K_{j^{\prime}}:=\left\{\left(j^{\prime}, k_{1}, k_{2}\right), 0 \leq k_{1}, k_{2} \leq 2^{j^{\prime}}-1\right\}$. Applying Fourier transform 2 , we have

$$
\hat{\theta}_{\alpha}^{\Omega}\left(w_{1}, w_{2}\right) \sim m_{j^{\prime}}\left(\theta_{\alpha}^{\Omega}\right)\left(w_{1}, w_{2}\right) \hat{\Phi}_{j^{\prime}, 0,0}^{\Omega}\left(w_{1}, w_{2}\right),
$$

where the symbol

$$
\begin{aligned}
m_{j^{\prime}}\left(\theta_{\alpha}^{\Omega}\right)\left(w_{1}, w_{2}\right) & =\sum_{\alpha^{\prime} \in K_{j^{\prime}}} C_{\alpha^{\prime}}^{\alpha} e^{-i 2 \pi\left(k_{1} w_{1}+k_{2} w_{2}\right) / 2^{j^{\prime}}} \\
& =4^{j^{\prime}} \sum_{z_{1}, z_{2} \in \mathbf{Z}} \hat{\theta}_{\alpha}^{\Omega}\left(w_{1}+2^{j^{\prime}} z_{1}, w_{2}+2^{j^{\prime}} z_{2}\right) \overline{\hat{\Phi}_{j^{\prime}, 0,0}^{\Omega}\left(w_{1}+2^{j^{\prime}} z_{1}, w_{2}+2^{j^{\prime}} z_{2}\right)} .
\end{aligned}
$$

Since the functions $\hat{\Phi}_{j^{\prime}, 0,0}^{\Omega}$ and $\left\{\hat{\theta}_{\alpha}^{\Omega}\right\}_{\alpha \in K_{j}}$ have exponential decay at infinity [24], the summation in index $z_{1}$ and $z_{2}$ is truncated to calculate $m_{j^{\prime}}\left(\theta_{\alpha}^{\Omega}\right)$. Thus the scaling coefficients $\left\{C_{\alpha^{\prime}}^{\alpha}\right\}_{\alpha^{\prime} \in K_{j^{\prime}}}$ are obtained by applying discrete inverse fourier transform on $m_{j^{\prime}}\left(\theta_{\alpha}^{\Omega}\right)$.

$$
{ }^{2} \forall f \in L^{2}\left(\mathbf{R}^{2}\right), \hat{f}\left(w_{1}, w_{2}\right)=\int_{\mathbf{R}^{2}} f(x, y) e^{-i 2 \pi\left(x w_{1}+y w_{2}\right)} d x d y
$$




\subsection{Calculation $C, D, F, G$ matrices}

We first consider the matrix F. In view of $\theta_{\alpha}^{\Omega}$ being approximated by a linear combination of scaling functions $\left\{\Phi_{\alpha^{\prime}}^{\Omega}\right\}_{\alpha^{\prime} \in K_{j^{\prime}}}$ in space $V_{j^{\prime}}$, we decompose the calculation of the elements of vector $F$ into $\int_{\Omega} \tilde{f} \Phi_{\alpha^{\prime}}^{\Omega}$. Assuming that the grid point values of $\tilde{f}$ are known at scale $\mathrm{j}$, we then use the interpolation process to approximate $\tilde{f}$,

$$
\tilde{f} \sim P_{V_{j}} \tilde{f}=\sum_{\alpha \in K_{j}} C_{\alpha} \Phi_{\alpha}^{\Omega}
$$

where $C_{\alpha}=\sum_{m, n=0}^{2^{j}-1} \tilde{f}\left(m / 2^{j}, n / 2^{j}\right) L_{j}\left(k_{1}-m\right) L_{j}\left(k_{2}-n\right)$. An explicit expression of $L_{j}$ is given in [25] when the spline multiresolution order $\mathrm{m}$ is even. In order to compute $\int_{\Omega} \Phi_{\alpha}^{\Omega} \Phi_{\alpha^{\prime}}^{\Omega}$, i.e., the scaling coefficients of $\Phi_{\alpha}^{\Omega}$ at scale $j^{\prime}$, we use the reconstruction algorithm.

Note that the elements of matrix C, D, G all involve the first form curve integral on $\Gamma$ or $\gamma$. We compute them similarly and detail the calculation of the matrix D for example. Let the parametric representation of curve $\gamma$ be

$$
\gamma:\left\{\begin{array}{l}
x=x(\tau) \\
y=y(\tau)
\end{array}, \quad \tau \in[0,1]\right.
$$

Thus we have

$$
D_{\alpha^{\prime \prime}, \alpha}=\int_{0}^{1} \Phi_{\alpha}^{\Omega}(x(\tau), y(\tau)) \phi_{\alpha^{\prime \prime}}^{[0,1]}(\tau) \sqrt{x^{\prime}(\tau)^{2}+y^{\prime}(\tau)^{2}} d \tau,
$$

where $\phi_{\alpha^{\prime \prime}}^{[0,1]}$ is the spline scaling function constructed on the interval $[0,1]$. $D_{\alpha^{\prime \prime}, \alpha}$ is its scaling coefficient with function $\Phi_{\alpha}^{\Omega} \sqrt{x^{\prime 2}+y^{\prime 2}}$. Thus $D_{\alpha^{\prime \prime}, \alpha}$ can be obtained by the interpolation process with the grid point values of $\Phi_{\alpha}^{\Omega} \sqrt{x^{\prime 2}+y^{\prime 2}}$ at scale $j^{\prime \prime}$. To improve its accuracy, we calculate the grid point values at upgraded scale $j^{\prime \prime}+\tilde{r}$ (for example, $\tilde{r}=3$ ), then obtain the scaling coefficients at scale $j^{\prime \prime}+\tilde{r}$ by the interpolation process. The decomposition algorithm is then employed to get the scaling coefficients at scale $j^{\prime \prime}$, i.e., $D_{\alpha^{\prime \prime}, \alpha}$.

\subsection{Saddle point system}

For the saddle point system $(5)$ where the $(1,1)$ block matrix I (identity matrix) is symmetric positive definite and the matrix $D C$ is nonsingular 
inferred from Lemma 2, we solve it by Schur complement reduction method $[15]$

$$
\left\{\begin{aligned}
D C \Lambda_{h^{\prime}} & =D F-G, \\
U_{h} & =F-C \Lambda_{h^{\prime}} .
\end{aligned}\right.
$$

The QMR method [21] is applied on the first equation to obtain $\Lambda_{h^{\prime}}$. Then $U_{h}$ follows by the second equation.

\section{Numerical examples}

To illustrate the improved accuracy order with the smooth fictitious domain/multiresolution method, we consider the problem $\hat{u}-\triangle \hat{u}=f$ on the domain $\omega$ represented by a cassini oval with boundary $\gamma$, i.e., $\gamma=\{(x, y)=$ $\left.(r \cos (2 \pi \tau), r \sin (2 \pi \tau)) \in \mathbf{R}^{2}, \tau \in[0,1], r=a \sqrt{\cos (4 \pi \tau)+\sqrt{(b / a)^{4}-\sin ^{2}(4 \pi \tau)}}\right\}$, where $a=0.25$ and $b=0.255$. The control boundary $\Gamma$ is also a cassini oval located outside $\gamma$ with distance 0.1 (see in Figure 2).

The extended enforcing function $\tilde{f}$ and boundary conditions lead to the exact solution $\hat{u}(x, y)=100\left((x-0.5)^{3}-(y-0.5)^{3}\right)-x^{2}$. The discretization levels on $\Omega, \Gamma$ and $\gamma$ are equal, i.e., $j=j^{\prime}=j^{\prime \prime}$. We choose the multiresolution order of $U_{h}^{\Omega}, m=4$. The figures 3,4 show the computed solution $u_{h}$ and the error $u_{h}-\hat{u}$ in $\omega$ with $j=j^{\prime}=j^{\prime \prime}=8$. In Table 1 , we compare the convergence rate of SFDLM and FDLM coupled with multiresolution discretization. It is clear that we obtain not only a smaller error, but also a higher accuracy order with SFDLM method which well preserves the high approximation ability of multiresolution methods.

Table 1: Comparison of convergence rate between SFDLM and FDLM

\begin{tabular}{ccccc}
\hline $\mathrm{j}$ & $\begin{array}{c}\operatorname{Err}_{L^{2}(\omega)} \\
(\mathrm{FDLM})\end{array}$ & $\begin{array}{c}\operatorname{Err}_{H^{1}(\omega)} \\
(\mathrm{FDLM})\end{array}$ & $\begin{array}{c}\operatorname{Err}_{L^{2}(\omega)} \\
(\mathrm{SFDLM})\end{array}$ & $\begin{array}{c}\operatorname{Err}_{H^{1}(\omega)} \\
(\mathrm{SFDLM})\end{array}$ \\
\hline 5 & $6.8889 \mathrm{e}-002$ & $3.6861 \mathrm{e}+000$ & $5.5934 \mathrm{e}-003$ & $5.4005 \mathrm{e}-001$ \\
6 & $3.2836 \mathrm{e}-002$ & $2.4729 \mathrm{e}+000$ & $4.4242 \mathrm{e}-004$ & $6.0724 \mathrm{e}-002$ \\
7 & $1.7381 \mathrm{e}-002$ & $1.8385 \mathrm{e}+000$ & $1.4280 \mathrm{e}-005$ & $3.1269 \mathrm{e}-003$ \\
8 & $8.5636 \mathrm{e}-003$ & $1.2992 \mathrm{e}+000$ & $8.4436 \mathrm{e}-007$ & $2.2511 \mathrm{e}-004$ \\
order & 1.003 & 0.502 & 4.231 & 3.743 \\
\hline
\end{tabular}




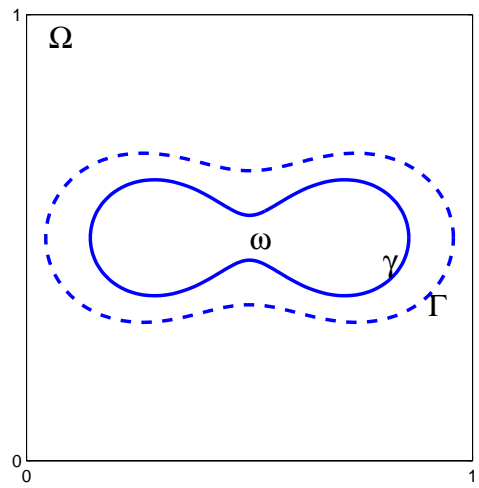

Figure 2: Geometry of $\omega$.

To observe the influence of the multiresolution order $\mathrm{m}$ on the error convergence rate, we use $\hat{u}(x, y)=\sin (2 \pi x)+\cos (2 \pi y), \mathrm{m}=4$ or 6 . Since the exact solution is periodic on fictitious domain $\Omega$, it follows that the Lagrange multiplier defined on control boundary $\Gamma$ is equal to 0 , i.e., $\bar{\lambda}=0$. The figures 5, 6 give the computed solution $u_{h}$ and the error $u_{h}-\hat{u}$ in $\omega$ with $j=j^{\prime}=j^{\prime \prime}=8, m=6$. Table 2 shows that the smooth fictitious domain/multiresolution method is of the orders of accuracy expected from Theorem 5 .

Table 2: Influence of multiresolution order $\mathrm{m}$ on convergence rate with SFDLM

\begin{tabular}{ccccc}
\hline $\mathrm{j}$ & $\begin{array}{c}\operatorname{Err}_{L^{2}(\omega)} \\
(\mathrm{m}=4)\end{array}$ & $\begin{array}{c}\operatorname{Err}_{H^{1}(\omega)}(\mathrm{m}=4) \\
\operatorname{Err}_{L^{2}(\omega)} \\
(\mathrm{m}=6)\end{array}$ & $\begin{array}{c}\operatorname{Err}_{H^{1}(\omega)} \\
(\mathrm{m}=6)\end{array}$ \\
\hline 5 & $6.4808 \mathrm{e}-007$ & $1.3154 \mathrm{e}-004$ & $7.2319 \mathrm{e}-008$ & $4.0914 \mathrm{e}-005$ \\
6 & $3.9516 \mathrm{e}-008$ & $1.8891 \mathrm{e}-005$ & $1.9038 \mathrm{e}-009$ & $1.0763 \mathrm{e}-006$ \\
7 & $2.5107 \mathrm{e}-009$ & $1.2732 \mathrm{e}-006$ & $7.0900 \mathrm{e}-012$ & $1.3329 \mathrm{e}-008$ \\
8 & $2.2054 \mathrm{e}-010$ & $1.2904 \mathrm{e}-007$ & $1.7128 \mathrm{e}-013$ & $2.7972 \mathrm{e}-010$ \\
order & 3.840 & 3.331 & 6.229 & 5.719 \\
\hline
\end{tabular}




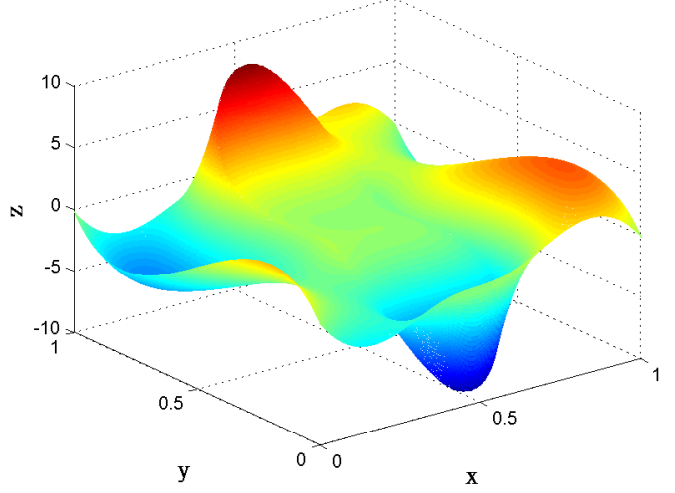

Figure 3: Computed solution $u_{h}$.

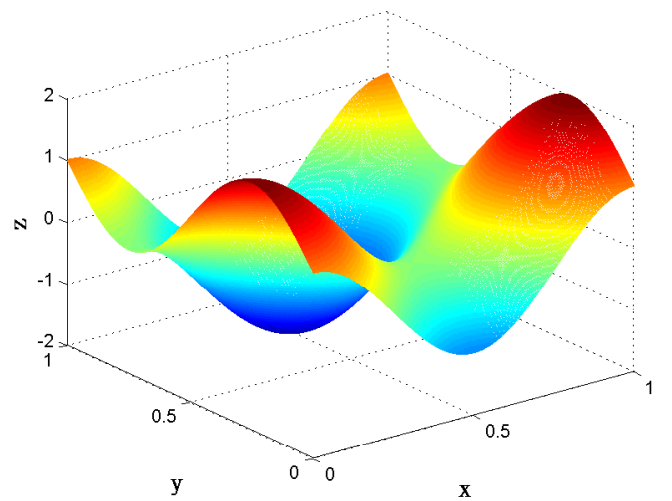

Figure 5: Computed solution $u_{h}$.

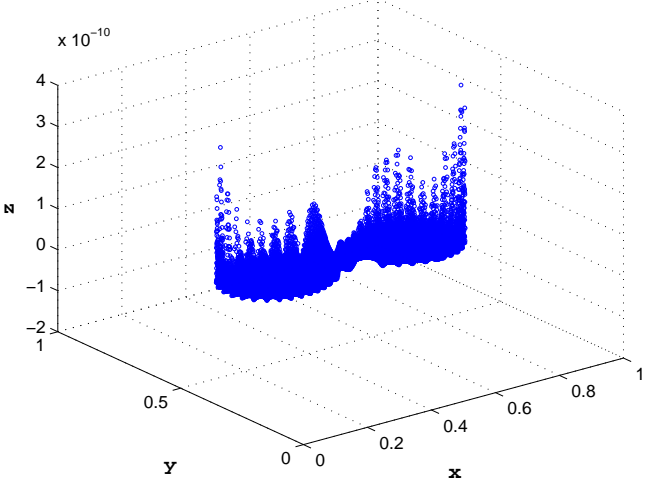

Figure 4: Error of $u_{h}-\hat{u}$.

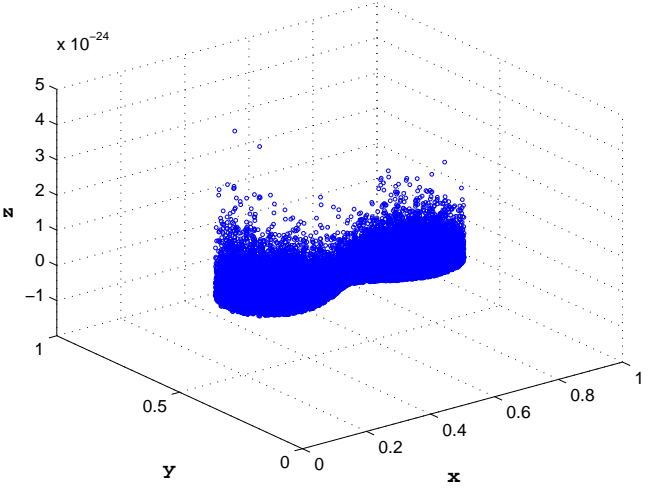

Figure 6: Error of $u_{h}-\hat{u}$. 


\section{Conclusions}

The main purpose of this short paper is to provide a numerical algorithm of high accuracy order for solving second order elliptic partial differential equations on general domain. We adopt the smooth fictitious domain/multiresolution method and prove interior error estimate. The numerical results confirm the theoretical analysis. Future work includes implementation of adaptive spaces of approximation, solution of problems of higher dimension and simulation of problems with moving boundary.

\section{Acknowledgement}

This work was supported by the National Natural Science Foundation of China(No.11226307), SRF for ROCS, SEM, the Natural Science Foundation of Jiangsu Province of China for Young Scholar (No. BK20130117).

\section{References}

[1] C. Burstedde, A. Kunoth, A wavelet-based nested iteration-inexact conjugate gradient algorithm for adaptively solving elliptic PDEs, Numer. Algorithms 48 (2008) 161-188.

[2] E. Deriaz., V. Perrier, Divergence-free and curl-free wavelets in two dimensions and three dimensions: application to turbulent flows, J. Turbul. 7 (2006) 1-37.

[3] P. Angot, C. Bruneau, P. Fabrie, A penalization method to take into account obstacles in incompressible viscous flow, Numer. Math. 81 (1999) 497-520.

[4] K. Schneider, M. Farge, Numerical simulation of the transient flow behaviour in tube bundles using a volume penalisation method, J. Fluid. Struct. 20 (2005) 555-566.

[5] L. Tomas, Optimisation de forme et domaines fictifs: analyse de nouvelles formulations et aspects algorithmiques, Ph.D. thesis, Ecole centrale de Lyon, 1997.

[6] M. S. Mommer, Towards a fictitious domain method with optimally smooth solutions, Ph.D. thesis, RWTH-Aachen, 2005. 
[7] J. Haslinger, T. Kozubek, R. Kucera, G. Peichl, Projected Schur complement method for solving non-symmetric systems arising from a smooth fictitious domain approach, Numer. Linear Algebra 14 (2007) 713-739.

[8] P. Yin, J. Liandrat, W. Q. Shen, Z. Chen, A multiresolution and smooth fictitious domain method for one-dimensional elliptical and Stefan problems, Math. Comput. Model. 58 (2013) 1727-1737.

[9] P. Yin, J. Liandrat, Coupling wavelets/vaguelets and smooth fictitious domain methods for elliptic problems: the univariate case, Comput. Appl. Math. (2014). DOI 10.1007/s40314-014-0136-9.

[10] A. Kunoth, Wavelet techniques for the Fictitious-Domain-LagrangeMultiplier-Approach, Numer. Algorithms 27 (2001) 291-316.

[11] F. Brezzi, M. Fortin, Mixed and Hybrid Finite Element Methods, Springer, 1991.

[12] L. C. Evans, Partial differential equations, American Mathematical Society, 1998.

[13] A. Quarteroni, A. Valli, Numerical approximation of partial differential equations, volume 23 of Springer series in computational mathematics, Springer-Verlag, 1997.

[14] R. Nicolaides, Existence,uniqueness and approximation for generalized saddle point problems, SIAM J. Numer. Anal. 19 (1982) 349-357.

[15] M. Benzi, G. Golub, J. Liesen, Numerical solution of saddle point systems, Acta Numerica (2005) 1-137.

[16] J. Haslinger, T. Kozubek, G. Peichl, A smooth embedding domain method based on the penalty approach, Num. Funct.Anal.and Optim. 32 (2011) 1252-1270.

[17] Y. Meyer, Ondelettes et Opérateurs I: Ondelettes, Hermann, 1990.

[18] J. Baccou, J. Liandrat, On coupling wavelets with fictitious domain approaches, Appl Math. Lett. 18 (2005) 1325-1331. 
[19] G. Chiavassa, J. Liandrat, A fully adaptive wavelet algorithm for parabolic partial differential equations, Appl. Numer. Math. 36 (2001) 333-358.

[20] A. Cohen, I. Daubechies, P. Vial, Wavelets on the interval and fast wavelet transforms, Appl. Comput. Harmon. Anal. 1 (1993) 54-81.

[21] G. H. Golub, C. F. V. Loan, Matrix computations, The Johns Hopkins University Press, 1996.

[22] J. Baccou, J. Liandrat, Definition and analysis of a wavelet/fictitious domain solver for the 2D-heat equation on a general domain, Math. Mod. Meth. Appl. S. 16 (2006) 819-845.

[23] W. Dahmen, A. Kunoth, Appending boundary conditions by Lagrange multipliers: Analysis of the LBB condition, Numer. Math. 88 (2002) 9-42.

[24] A. Cohen, Wavelets methods in numerical analysis, handbook of numerical analysis, North-Holland, 2000.

[25] V. Perrier, C. Basdevant, La décomposition en ondelettes périodiques, un outil pour l'analyse de champs inhomogènes. théorie et algorithmes, La recherche aerospatiale 3 (1989) 57-67. 